\title{
On the Uniqueness of Static Perfect-Fluid Solutions in General Relativity *
}

\author{
R. Beig and W. Simon \\ Institut für Theoretische Physik, Universität Wien, A-1090 Wien, Austria
}

Received August 20, 1990

\begin{abstract}
Following earlier work of Masood-ul-Alam, we consider a uniqueness problem for non-rotating stellar models. Given a static, asymptotically flat perfectfluid spacetime with barotropic equation of state $\varrho(p)$, and given another such spacetime which is spherically symmetric and has the same $\varrho(p)$ and the same surface potential: we prove that both are identical provided $\varrho(p)$ satisfies a certain differential inequality. This inequality is more natural and less restrictive than the conditions required by Masood-ul-Alam.
\end{abstract}

\section{Introduction}

In this paper we solve a uniqueness problem for non-rotating stellar models. Namely, given a static, asymptotically flat perfect-fluid spacetime with barotropic equation of state $\varrho(p)$ subject to certain restrictions: we show that this is unique provided there exists another such model [called "spherical reference (SR-) model"] which is spherically symmetric and has the same $\varrho(p)$ and the same value $V_{s}$ of the gravitational potential on the boundary of the star ("surface redshift"). This problem was first raised and solved by Masood-ul-Alam [1] under more restrictive and somewhat unnatural conditions on $\varrho(p)$.

The main motivation for the present problem comes from the "fluid ball conjecture" which states that a non-rotating stellar model is spherically symmetric (see Künzle and Savage [2]). If this was known, it would follow that such a model consists of a family of solutions determined by one parameter, e.g. the value of the pressure at the centre. (For a rigorous proof of this fact see Schmidt [3].) In the absence of a direct proof of spherical symmetry for general $\varrho(p)$, one tries to turn this reasoning around by concluding sphericity from uniqueness for some given parameter, and here the surface redshift turns out to be a possible choice. However this parametrization involves a problem, as can be seen already in the spherical case: Consider, instead of $V_{s}$, the mass $m$ of a spherically symmetric configuration as a function of the central pressure $p_{c}$. For degenerate matter $m\left(p_{c}\right)$ is well known

* Supported by Fonds zur Förderung der wissenschaftlichen Forschung in Österreich, project P-7197 
[4] to be increasing when $p_{c}$ is not too large, but it develops a local maximum which corresponds to the onset of gravitational instability. As the numerical results show $[5,6]$ a similar thing happens with $V_{s}\left(p_{c}\right)$, although typically at larger values of $p_{c}$. Thus $p_{c}$ will in general be a multi-valued function of $V_{s}$. If arbitrary large values of $p_{c}$ were allowed (ignoring, of course, that nothing is known about the physics in this regime) the results of Harrison et al. (see [5, Sect. 5]) show that for equations of state $\varrho(p)$ which are asymptotically linear for large $p, m_{\infty}=\lim _{p \rightarrow \infty} m\left(p_{c}\right)$ and $V_{\infty}=\lim _{p \rightarrow \infty} V_{s}\left(p_{c}\right)$ exist and are in fact infinitely degenerate.

Thus the uniqueness-problem studied here will necessarily entail some bound on $\varrho(p)$ - or on the range of $p$ for given $\varrho(p)$ - which rules out the multi-valuedness of $p_{c}\left(V_{s}\right)$. It remains a mathematical challenge to prove the fluid-ball conjecture without such a restriction, i.e. independently of stability questions. On the other hand it is precisely this relationship with stability which endows the uniqueness problem studied here with independent interest.

We now sketch Masood-ul-Alam's approach and our improvement thereof, where such an additional condition on $\varrho(p)$ emerges in an apparently natural fashion. The main tool is a corollary to the positive-mass theorem. This states that a complete, asymptotically flat Riemannian metric must be flat if its Ricci scalar is everywhere non-negative, and if its mass vanishes [7]. To explain the use of this theorem in the present problem, we first recall (a part of) the uniqueness proof for non-rotating black holes due to Bunting and Masood-ul-Alam [8]. When $d s^{2}$ $=-V^{2} d t^{2}+g_{a b} d x^{a} d x^{b}$ is the static spacetime metric, these authors use a conformal rescaling of the form $\hat{g}_{a b}=\frac{1}{16}(1+V)^{4} g_{a b}$. Because of the field equations, $\hat{g}_{a b}$ has zero mass and zero scalar curvature. This metric can be extended through the horizon to a complete Riemannian metric with everywhere vanishing Ricci scalar. Thus, by the above-mentioned theorem, $\hat{g}_{a b}$ is flat. The rest follows by an easy integration of the static field equations.

One now tries to do the same thing for perfect fluids, namely to conformally rescale the metric such that it meets again the requirements of the positive mass theorem. We first note that this can be accomplished straightforwardly if $\varrho(p)$ falls under the one-parameter family of equations of state $p(\varrho)=\frac{1}{6} \varrho^{6 / 5}\left(\varrho_{0}^{1 / 5}-\varrho^{1 / 5}\right)^{-1}$, $\varrho<\varrho_{0}=$ const $>0$, since for such solutions we again have $R[\hat{g}]=0$ everywhere, where $\hat{g}_{a b}=\frac{1}{16}(1+V)^{4} g_{a b}$ as before. For any $\varrho_{0}>0$, one finds [9] that $\left(g_{a b}, V\right)$ is a member of a one-parameter family of solutions given by Buchdahl [10]. We remark that for all these solutions the matter region necessarily extends to infinity.

In order to determine a suitable conformal transformation for general - i.e. functionally unrestricted - equations of state, one invokes the existence of a spherical reference solution $\left({ }^{0} g_{a b},{ }^{0} V\right)$. We take $\Omega(V)$ to be ${ }^{0} \Omega(V)$, where ${ }^{0} \Omega$ is chosen such that $R\left[\left({ }^{0} \Omega\right)^{2}{ }^{2} \mathrm{~g}\right]=0$. In particular, $\Omega=\frac{1}{4}(1+V)^{2}$ in the vacuum region. In the fluid region, $R\left[\Omega^{2} g\right]$ takes on the form $R\left[\Omega^{2} g\right]=P(V)\left[W_{0}(V)-W\right]$, where $W=g^{a b} D_{a} V D_{b} V$ and $W_{0}\left({ }^{\circ} V\right)={ }^{0} g^{a b} D_{a}^{0} V D_{b}^{0} V$. To ensure the required non-negativity of $R\left[\Omega^{2} g\right]$ it has proved useful (and seems to be necessary) to keep control over the signs of each of its factors separately. It was shown in [1] that $P(V) \geqq 0$ provided $\varrho(p)$ satisfies $K \leqq 0$, where

$$
K=p(\varrho+p) \frac{d \varrho}{d p}-\frac{5}{6} \varrho^{2} .
$$

Furthermore $P(V)=0$ iff $K=0$ which just leads to the Buchdahl solutions discussed before. 
It remains to estimate $W-W_{0}$. The general idea is to derive, from the field equations for $\left(V, g_{a b}\right)$ and for $\left({ }^{0} V,{ }^{0} g_{a b}\right)$, an elliptic equation for this quantity. If this equation takes the form

$$
\left(g^{a b} \partial_{a} \partial_{b}+k^{a} \partial_{a}+c\right)\left(W-W_{0}\right) \geqq 0, \text { with } c \leqq 0,
$$

the maximum principle is applicable which, taken together with boundary conditions, yields the desired result. Such identities were found first in the vacuum case for the purpose of uniqueness-proofs for static black holes [11,12] (under more restrictive conditions than [8]), and in the case of perfect fluids of constant density $[13,14]$. In [1] Masood-ul-Alam uses a somewhat ad-hoc-modification of these identities and shows that $c \leqq 0$ under rather restrictive conditions on the equation of state.

In the present work, we find an identity of the form $(\tilde{\Delta}+c)\left(\tilde{W}-\tilde{W}_{0}\right) \geqq 0$. Here $\tilde{\Delta}$ is formed from the metric $\widetilde{g_{a b}}=V^{-2} \Psi^{4} g_{a b}$ and $\tilde{W}-\widetilde{W}_{0}=\Psi^{-4}\left(W-W_{0}\right)$ where, for given $\varrho(p)$, the quantity $\Psi(V)$ is determined by an ordinary first-order differential equation. In the vacuum case, and in the case of a fluid of constant density, this identity [with $\Psi=\frac{1}{2}\left(1-V^{2}\right)$ and $\Psi=1$ respectively, and with $c=0$ in either case] reduces to ones used by Robinson [12] and Lindblom [13]. Equations of state satisfying $K=0$ are again special in that one obtains $\Psi=\frac{1}{2}\left(1-V^{2}\right)$ and $c=0$, exactly as in vacuum. In this case application of the maximum principle to $\widetilde{\Delta}\left(\tilde{W}-\tilde{W}_{0}\right) \geqq 0$ provides an alternative proof of uniqueness of the Buchdahl solutions [9]. In general, we use our identity inside as well as outside the star provided the density $\varrho$ goes to zero at the boundary. (If this is not the case we have to take, in the vacuum region, some other element of the two-parameter family given in [12]). The non-positivity of the coefficient $c$ is ensured if $I \leqq 0$ where

$$
I=\frac{1}{5} \kappa^{2}+2 \kappa+(\varrho+p) \frac{d \kappa}{d p}
$$

with $\kappa=(\varrho+p)(\varrho+3 p)^{-1} d \varrho / d p$.

The remarkable feature of this condition is the following. Although neither the factors $P(V)$ and $W-W_{0}$ in $R\left[\Omega^{2} g\right]$ themselves, nor the ways of estimating them, seem to have anything in common, surprisingly, the quantities $K$ and $I$ which enter their respective estimates, are related. We can show that $I \leqq 0$ implies $K \leqq 0$. Conversely, $K=0$ implies $I=0$. Thus, if we only assume $I \leqq 0$, the maximum principle combined with the positive-mass theorem shows that $\Omega^{2} g_{a b}$ is flat which in turn implies that $\left(g_{a b}, V\right)$ is spherically symmetric. To prove uniqueness one has to further conclude $W=W_{0}$ which was apparently overlooked in [1]. Doing this turns out to be equivalent to ruling out the Buchdahl case $K=0$.

To get an idea of how restrictive the condition $I \leqq 0$ is, one can check it for an ideal fermion gas at zero temperature. One finds that $I \leqq 0$ is valid for densities up to $4 \times 10^{15} \mathrm{gcm}^{-3}$ which is roughly the critical density where gravitational instability sets in [15]. We note that for the more realistic models of neutron stars governed by the Harrison-Wheeler equation of state (see [5, Sect. 10]) $I \leqq 0$ is violated in regions where neutron drip occurs.

Finally, we would like to mention two problems which one has to overcome in the proof. Firstly, the minimal values for $V$ of the given star and of the SR-model, and hence the domains of $W$ and $W_{0}$, need not agree. One can, however, continue $W_{0}(V)$ into the region $V<{ }^{0} V_{\min }$, which we do in a manner different from Masoodul-Alam. Another problem is that, if the density $\varrho$ goes to zero at the boundary of 
the star, the coefficient $c$ introduced before may diverge there. This is not allowed in the Hopf boundary-point lemma [16] which we need in the proof. We found, however, an extension of this lemma which copes with the present kind of singularity, and which could be of independent interest.

The present paper is organized as follows. In Sect. 2 we define what we mean by a static perfect-fluid model and obtain some easy consequences. In Sect. 3 we discuss a few properties, mostly known, of the spherical case and prove a uniqueness lemma, similar to an early result of Künzle [17] (see also Avez [18]). In Sect. 4 we introduce the inequality $I \leqq 0$ and derive the consequences $K \leqq 0$ and $c \leqq 0$. Section 5 contains the main part of the uniqueness proof. In Sect. 6 we discuss the Newtonian situation and possible improvements of our results. Finally, the Appendix deals with the above-mentioned extension of the boundary-point lemma.

\section{General Assumptions and Properties}

We introduce a static metric $d s^{2}=-V^{2} d t^{2}+g_{a b} d x^{a} d x^{b}(V>0)$, where both the Riemannian metric $g_{a b}$ and the potential $V$ are (time-independent) functions on a 3-manifold $M$. We assume for simplicity that there is only one body, leaving a remark on the $n$-body case to the end of Sect. 5. More precisely we assume that there is an open, connected set $Q \subset M$ with smooth boundary $\partial Q \subset M$ and $\bar{Q}=Q \cup \partial Q$ compact. We also assume $\partial Q$ to be connected, thus excluding the possibility of a "hollow" body. It follows that $E=M \backslash \bar{Q}$ is also connected. There are functions $\varrho$ and $p$ on $M$ which are zero in $E$ and smooth and positive in $Q$. Furthermore, on $\partial Q, p$ is zero and $V$ takes the constant value $V_{s}$. The fields $g_{a b}$ and $V$ are smooth both in $Q$ and $E$ and satisfy the Lichnerowicz junction conditions [19]

$$
g_{a b}, V \in C^{1}(M) \cap C^{2}(\bar{Q}) \cap C^{2}(\bar{E}) .
$$

They obey Einstein's static equations with a perfect-fluid source of density $\varrho$ and pressure $p$, i.e.

$$
\begin{gathered}
R_{a b}=V^{-1} D_{a} D_{b} V+4 \pi(\varrho-p) g_{a b}, \\
\Delta V=4 \pi V(\varrho+3 p),
\end{gathered}
$$

where the covariant derivative $D_{a}, \Delta=D^{a} D_{a}$ and the Ricci tensor $R_{a b}$ are formed from $g_{a b}$.

We also assume that there is an equation of state of the form $\varrho=\varrho(p)$, with $d \varrho / d p \geqq 0$, where $\varrho(p)$ is smooth in an interval $\left[0, p_{\max }\right]$ when $\varrho(0)>0$. When $\varrho(0)$ is zero, in order for $p(V)$ to exist, we have in addition to require that $\int_{0}^{p}[\varrho(s)+s]^{-1} d s<\infty$ for finite $p$. This implies $\lim _{p \rightarrow 0} d \varrho / d p=\infty$. Thus, in the case $\varrho(0)=0$, we just demand smoothness of $\varrho(p)$ in $\left(0, p_{\max }\right]$.

The global conditions on $M$ are as follows: After adding a point, the "point-atinfinity" $\Lambda, \tilde{M}=M \cup\{\Lambda\}$ becomes a compact manifold without boundary. At $\Lambda$, we have the following asymptotic conditions: $V$ extends to a $C^{0}$-function on $\tilde{M}$ with $\left.V\right|_{\Lambda}=1$. Furthermore $\check{g}_{a b}=\frac{1}{16}(1-V)^{4} g_{a b}$ and $\sigma=(1-V)^{2}(1+V)^{-2}$ extend respectively to a smooth metric and a smooth function on $\tilde{M} \backslash \bar{Q}$ with $\left.\sigma\right|_{A}=\left.D_{a} \sigma\right|_{A}=0$. One can show $[20,21]$ that these conditions imply that the quantity $\sigma^{-1} g^{a b} D_{a} \sigma D_{b} \sigma$ is $C^{\infty}$ at $\Lambda$. 
We remark that these asymptotic conditions imply the usual ones: one easily finds that there exists a coordinate system $x^{a}$ in a punctured neighbourhood of $\Lambda$, such that

$$
g_{a b}-\delta_{a b}=O^{\infty}\left(r^{-1}\right), \quad V-1=O^{\infty}\left(r^{-1}\right),
$$

where $r^{2}=\delta_{a b} x^{a} x^{b}$ and $f=O^{\infty}\left(r^{-1}\right)$ means $f=O\left(r^{-1}\right), \partial f=O\left(r^{-2}\right)$, a.s.o. It is less obvious but follows from the field equations [22], that the converse is also true. In fact, given Eqs. $(2.2,2.3) \check{g}_{a b}$ and $\sigma$ are even analytic near $\Lambda$.

In the sequel the notion of a "static perfect-fluid model" will refer to a solution $\left(M, g_{a b}, V\right)$ satisfying the above conditions. We now point out some of their consequences.

Lemma 1. $V_{s}<V<1$ in $E$ and $V_{\min } \leqq V<V_{s}$ in $Q$.

Proof. The lemma follows easily by applying the maximum principle (see Theorem 3.5 in [16]) to Eq. (2.3) on the domains $Q$ and $E$ separately. Possible maxima of $V$ on $\partial Q$ are excluded by virtue of the boundary point lemma (Lemma (3.4) in [16]).

Another elementary but important fact is contained in

Lemma 2. The pressure $p$ is a monotonically decreasing function of $V$ satisfying

$$
\frac{d p}{d V}=-V^{-1}(\varrho+p)
$$

or, equivalently,

$$
G(V) \equiv V^{-1} V_{s} \exp \left\{-\int_{0}^{p}[\varrho(s)+s]^{-1} d s\right\}=1 .
$$

Proof. Applying the Bianchi identity $D^{a}\left(R_{a b}-\frac{1}{2} g_{a b} R\right)=0$ to $(2.2,2.3)$ we find

$$
D_{a} p=-V^{-1}(\varrho+p) D_{a} V
$$

and hence $D_{a} G=0$ in $Q$. Thus, since $Q$ is connected, $G$ is constant. But since $G=1$ on $\partial Q$, Eq. (2.6) follows.

The point of this lemma is that one does not need any assumptions concerning the critical set of $V$. We remark that since $d \varrho / d p \geqq 0, \varrho$ is also a function of $V$ which is uniquely determined by $V_{s}$ and satisfies $d \varrho / d V \leqq 0$. In the sequel we use for $\varrho$ on $\partial Q$ interchangeably the forms $\varrho(0)$ or $\varrho(V)$, depending on whether we consider $\varrho$ as a function of $p$ or as a function of $V$.

We finally note an important identity due to Lindblom [13].

Lemma 3. With the definitions

$$
W=D_{a} V D^{a} V
$$

and the "Cotton tensor"

$$
B_{a b c}=2 D_{[c}\left(R_{b] a}-\frac{1}{4} g_{b] a} R\right)
$$

there holds

$$
\begin{aligned}
\Delta W= & \frac{1}{4} V^{4} W^{-1} B_{a b c} B^{a b c}+V^{-1} D^{a} V D_{a} W+8 \pi V D^{a} V D_{a} \varrho+\frac{3}{4} W^{-1} D^{a} W D_{a} W \\
& -8 \pi W(\varrho+p)+16 \pi^{2} V^{2}(\varrho+3 p)^{2}-4 \pi V(\varrho+3 p) W^{-1} D^{a} V D_{a} W .
\end{aligned}
$$


for all non-critical values of $V$. The proof is by direct computation, using Eq. $(2.2,2.3)$.

The function $W$, from (2.1), is $C^{0}$ across $\partial Q$. It satisfies [13]

$$
\left(W^{-1} D^{a} V D_{a} W\right)\left(V_{s}^{>}\right)-\left(W^{-1} D^{a} V D_{a} W\right)\left(V_{s}^{<}\right)=-8 \pi V_{s} \varrho(0),
$$

where ( $)\left(V_{s}^{>}\right)$and ()$\left(V_{s}^{<}\right)$denote the limits $V \rightarrow V_{s}$ of the quantities in brackets for $V>V_{s}$ and $V<V_{s}$ respectively. Equation (2.11) follows from decomposing Eq. (2.3) as

$$
W^{-1} D^{a} V D_{a} W=-2 W^{1 / 2} k+8 \pi V(\varrho+3 p),
$$

where $k$ is the mean curvature of the $V$-level sets.

\section{The Spherical Case}

We now discuss some properties of a static perfect-fluid model $\left(M, g_{a b}, V\right)$ which is in addition spherically symmetric. Since $\partial Q$ is by assumption connected it follows in this case that $Q$ is a ball and $M \cong \mathbf{R}^{3}$. The metric can be brought into the form

$$
g=-V^{2} d t^{2}+h d r^{2}+r^{2} d \omega^{2}
$$

where $d \omega^{2}=d \vartheta^{2}+\sin ^{2} \vartheta d \varphi^{2}$ and $V$ and $h$ are smooth functions of $r^{2}$ for $r \leqq r_{s}$. Explicitly we have

$$
h(r)=\left(1-2 r^{-1} m(r)\right)^{-1}
$$

for $r \leqq r_{s}$, where $m(r)=4 \pi \int_{0}^{r} \varrho\left(r^{\prime}\right) r^{\prime 2} d r^{\prime}$ and

$$
h(r)=\left(1-2 r^{-1} m\right)^{-1}, \quad V(r)=\left(1-2 r^{-1} m\right)^{1 / 2}
$$

for $r>r_{s}$, where $m=m\left(r_{s}\right)$. There is in general no explicit form of $V(r)$ for $r \leqq r_{s}$ but, as we shall see presently, $V(r)$ is implicitly determined by $W$. It follows from the maximum principle that $d V / d\left(r^{2}\right)>0$ for $0 \leqq r \leqq r_{s}$. Thus $r^{2}$ can be considered as a smooth function in $\left[V_{c}, V_{s}\right)$ with $r^{2}\left(V_{c}\right)=0$ and $r^{2}\left(V_{s}\right)=r_{s}^{2}$. Hence $W$ is also a smooth function in $\left[V_{c}, V_{s}\right)$, which for later convenience we write as $W_{0}(V)$. We note that $W_{0}\left(V_{c}\right)=0$ and $W_{0}>0$ in $\left(V_{c}, V_{s}\right]$.

Specializing Eq. (2.10) to the spherical case, where $B_{a b c}=0$ and $g=W_{0}^{-1} d V^{2}$ $+r^{2}(V) d \omega^{2}$ for $V>V_{c}$, we find

$$
\begin{aligned}
W_{0} W_{0}^{\prime \prime}= & \frac{3}{4}\left(W_{0}^{\prime}\right)^{2}+\left[V^{-1} W_{0}-8 \pi V(\varrho+3 p)\right] W_{0}^{\prime} \\
& +8 \pi V W_{0}(\varrho+p)^{\prime}+16 \pi^{2} V^{2}(\varrho+3 p)^{2},
\end{aligned}
$$

where primes denote derivatives with respect to $V$. Evaluating Eq. (3.4) at $V=V_{c}$, we find that one of the quantities

$$
L_{1 / 3}(V)=W_{0}^{\prime}-\frac{8 \pi}{3} V(\varrho+3 p)
$$

or

$$
L_{1}(V)=W_{0}^{\prime}-8 \pi V(\varrho+3 p)
$$


must vanish at $V=V_{c}$. In the spherical case, Eq. (2.12) becomes

$$
W_{0}^{\prime}=-2 W_{0}^{1 / 2} k+8 \pi V(\varrho+3 p)
$$

and $k=k(V)$ is given by

$$
k=\frac{1}{2} W_{0}^{1 / 2} r^{-2}\left(r^{2}\right)^{\prime} .
$$

If $L_{1}\left(V_{c}\right)=0$ then $k\left(V_{c}\right)$ would be zero and so $r=0$ would not be a regular point. Thus $L_{1 / 3}\left(V_{c}\right)=0$ holds. Dividing Eq. (3.4) by $W_{0}$ and taking the limit $V \rightarrow V_{c}$, we find

$$
W_{0}^{\prime \prime}\left(V_{c}\right)=-\frac{16 \pi}{3} \varrho\left(V_{c}\right)+\frac{24 \pi}{5} V_{c} \varrho^{\prime}\left(V_{c}\right) .
$$

In the exterior region, $W_{0}(V)$ is explicitly given by

$$
W_{0}(V)=\frac{1}{16} m^{-2}\left(1-V^{2}\right)^{4} .
$$

Using (2.11) and (3.10), it follows that

$$
W_{0}^{\prime}\left(V_{s}^{<}\right)+8 V_{s}\left(1-V_{s}^{2}\right)^{-1} W_{0}\left(V_{s}\right)=8 \pi V_{s} \varrho\left(V_{s}\right) .
$$

We shall need the following uniqueness lemma.

Lemma 4 (Compare Avez [18], Künzle [17]). Let there be given a model $\left(M, g_{a b}, V\right)$, not a priori spherical, where $W=D_{a} V D^{a} V$ is a given function $W_{0}$ of $V$ which is positive for $V>V_{\min }$, smooth in $\left[V_{\min }, V_{s}\right)$ and satisfies $W_{0}\left(V_{\min }\right)=0$ and $L_{1}\left(V_{\min }\right) \neq 0$, where $L_{1}(V)$ is given by $E q$. (3.6). Then $\left(M, g_{a b}, V\right)$ is a spherically symmetric solution uniquely determined by $W_{0}(V)$.

Proof. Using $W=W_{0}(V)$ in Eq. (2.12) we conclude that $k=k(V)$. Near infinity, the level sets of $V$ are 2-spheres. Since, by assumption, $V_{\min }$ is the only critical value of $V$, the surfaces $V=$ const form a family of nested 2-spheres for all $V_{\min }<V<1$. In the case where $W=W_{0}(V)$ and $k=k(V)$, the field equation (2.2) shows that

$$
D^{A}\left(k_{A B}-\frac{k}{2} \gamma_{A B}\right)=D^{A} k_{A B}=0,
$$

where $\gamma_{A B}$ and $k_{A B}$ are the metric and the extrinsic curvature on $V=$ const, and $D_{A}$ is the covariant derivative w.r.t. $\gamma_{A B}$. Since $\gamma_{A B}$ is conformal to the unit 2-sphere, this implies (see, e.g. [23])

$$
\mathscr{L}_{\Gamma} \gamma_{A B} \equiv 2 W_{0}^{-1} k_{A B}=W_{0}^{-1} k \gamma_{A B},
$$

where $\mathscr{L}_{\Gamma}$ denotes the Lie-derivative w.r.t. to $\Gamma^{a}=W_{0}^{-1} D^{a} V$. We now choose a coordinate system $(\vartheta, \varphi)$ on some initial 2-sphere such that

$$
\gamma_{A B} d x^{A} d x^{B}=r^{2}\left(d \vartheta^{2}+\sin ^{2} \vartheta d \varphi^{2}\right)
$$

and extend it by $\mathscr{L}_{\Gamma} \vartheta=\mathscr{L}_{\Gamma} \varphi=0$. We find from Eq. (3.13) that the form of Eq. (3.14) holds for all values $V>V_{\min }$, where $r^{2}(V)$, from Eq. (3.8), satisfies

$$
r^{-2}\left(r^{2}\right)^{\prime}=\left(2 W_{0}\right)^{-1} L_{1}(V) .
$$

Note that $W_{0}^{-1} L_{1}$ is $C^{0}$ at $V=V_{\min }$. It follows that $g_{a b}$ is locally spherically symmetric so that Eq. (3.4) holds. This, in turn, implies

$$
W_{0} L_{1}^{\prime}=\frac{3}{4} L_{1}^{2}+\left[V^{-1} W_{0}+4 \pi V(\varrho+3 p)\right] L_{1}+16 \pi W_{0}(\varrho+p) .
$$


We claim that $L_{1}(V)<0$ for $V<1$. This is clear for $V>V_{s}$, where $W_{0}(V)$ is of the form of Eq. (3.10). For $V<V_{s}$ we can argue as follows: Eq. (2.11) means that $L_{1}(V)$ is continuous across $\partial Q$. Thus $L_{1}\left(V_{s}^{<}\right)<0$. If $L_{1}$ was zero for some $\bar{V}$, Eq. (3.16) would imply $L_{1}^{\prime}(\bar{V})>0$ which is a contradiction.

It follows that Eq. (3.15) defines a monotonically increasing, for $V>V_{\min }$ positive function $r^{2}(V)$. Using Eq. (3.16), Eq. (3.15) can be explicitly integrated to give

$$
r^{2}(V)=C^{2}\left[\left(16 W_{0}\right)^{-1} L_{1}^{2}-(2 V)^{-1} L_{1}-8 \pi p\right]^{-1},
$$

where $C$ is a constant. This shows that $r^{2}\left(V_{\min }\right)=0$ and that $r^{2}(V)$ is smooth in $\left[V_{\min }, V_{s}\right.$ ). Inverting Eq. (3.17) gives $V$ as a function of $r$. Observe, finally, that $h=W_{0}^{-1}(d V / d r)^{2}$. Since, by asymptotic flatness, $\lim _{r \rightarrow \infty} h(r)=1$, we have to set $C^{2}=1$, and so $h(r)$ and $V(r)$ are determined. Thus $\left(g_{a b}, V\right) \stackrel{r \rightarrow \infty}{\text { is a }}$ unique spherically symmetric solution which completes the proof.

\section{The Condition $I \leq 0$}

In the introduction, we defined the quantities

$$
K=6 p(\varrho+p) \frac{d \varrho}{d p}-5 \varrho^{2}
$$

and

$$
I=\frac{1}{5} \kappa^{2}+2 \kappa+(\varrho+p) \frac{d \kappa}{d p},
$$

where $\kappa=(\varrho+p)(\varrho+3 p)^{-1} d \varrho / d p$ and stated some of their properties which we prove here. The lemmas of this section require, as usual, $\varrho \geqq 0, p \geqq 0$, and $d \varrho / d p \geqq 0$. It is understood that, in the case $\varrho(0)=0, K$ and $I$ are defined only for $p>0$.

Lemma 5. $I \leqq 0$ implies $K \leqq 0$.

Proof. Define the quantity $\Phi(p)$ by

$$
\Phi(p)=\frac{1}{6}(\varrho+3 p)^{-1} p^{1 / 6} K=p^{7 / 6} \kappa-\frac{5}{6}(\varrho+3 p)^{-1} \varrho^{2} p^{1 / 6}
$$

for $p>0$. We first show that $\Phi(p)$ can be continuously extended to $p=0$. It is obvious that $\Phi(0)=0$ in the case where $\varrho(0)>0(d \varrho / d p$ finite). When $\varrho(0)=0$ and $d \varrho / d p$ is infinite, we first note that, since $I \leqq 0, \lambda=\kappa^{-1}$ satisfies

$$
(\varrho+p) \frac{d \lambda}{d p} \geqq \frac{1}{5}+2 \lambda,
$$

and hence $\lim _{p \rightarrow 0} d \lambda / d p=\infty$. Thus,

$$
\lim _{p \rightarrow 0} p \kappa=\lim _{p \rightarrow 0} \frac{p}{\lambda}=\lim _{p \rightarrow 0}\left(\frac{d \lambda}{d p}\right)^{-1}=0
$$

which, when used in Eq. (4.3), shows that $\Phi(0)=0$ in this case also.

By a long computation, one now finds

$$
(\varrho+p) \frac{d \Phi}{d p}=p^{7 / 6} I-\Phi\left[\frac{1}{5} p^{-7 / 6} \Phi+(\varrho+3 p)^{-1}\left(\frac{7}{3} \varrho+\frac{5}{2} p\right)\right]
$$


for $p>0$. If $\Phi$ were positive for some $\bar{p}>0$ we would obtain $(d \Phi / d p)(\bar{p})<0$ from Eq. (4.6) which leads to a contradiction with $\Phi(0)=0$. Hence $\Phi \leqq 0$ which proves the lemma.

We remark that, from Eq. (4.6) $K=0$ implies $I=0$. The converse is false, as the example $\varrho=$ const shows.

The conditions $I \leqq 0$ and $K \leqq 0$ imply estimates for the spherical case which will be crucial for the proof of our main theorem in the next section. One of these is contained in [1] and is recorded here for convenience.

Let $\left(g_{a b}, V\right)$ be spherical and $\Omega(V)$ be the (positive) function which makes $\Omega^{2} g$ Euclidean. $\Omega(V)$ is $C^{1}$ across $\partial Q$ and $d^{2} \Omega / d V^{2}$ makes a finite jump there. In the vacuum region $\left(V \geqq V_{s}\right)$ one can take

$$
\Omega(\mathrm{V})=\frac{1}{4}(1+\mathrm{V})^{2} .
$$

In $Q, \Omega(V)$ satisfies

$$
2 \Omega^{-1} \Omega^{\prime \prime}-\Omega^{-2}\left(\Omega^{\prime}\right)^{2}=\left[8 \pi \varrho-8 \pi V(\varrho+3 p) \Omega^{-1} \Omega^{\prime}\right] W_{0}^{-1} \equiv s W_{0}^{-1} .
$$

From [1] we take over the following result.

Lemma 6. $K \leqq 0$ implies $s \geqq 0$.

A quantity of prime importance is $\Sigma(V)$ defined by

$$
\Sigma(V)=L_{1 / 3}+\frac{4}{5} V^{-1} W_{0} \kappa .
$$

From $L_{1 / 3}\left(V_{c}\right)=0$ and from Eq. (3.9) we obtain $\Sigma\left(V_{c}\right)=\Sigma^{\prime}\left(V_{c}\right)=0$. There holds the following estimate for $\Sigma(V)$ :

Lemma 7. $I \leqq 0$ implies $\Sigma \geqq 0$ in $\left[V_{c}, V_{s}\right)$.

Proof. Equation (3.4) implies

$$
W_{0}^{3 / 2} t^{\prime}=\frac{1}{2} W_{0} V t^{2}-\frac{16 \pi}{3} V W_{0}^{1 / 2}(\varrho+3 p) t-\frac{2}{5} V^{-3} W_{0}^{2} I
$$

for the quantity $t(V)=\frac{1}{2} W_{0}^{-1 / 2} V^{-1} \Sigma$ in the domain $V \geqq V_{c}$. From l'Hospital's rule, we obtain $t\left(V_{c}\right)=0$. If $t$ was negative for some $\bar{V}>V_{c}$, then, by Eq. (4.10), $t^{\prime}(\bar{V})>0$ which is a contradiction. Thus $t(V) \geqq 0$ and hence $\Sigma(V) \geqq 0$.

We remark that, using $d \varrho / d p \geqq 0$, one can show that $L_{1 / 3} \leqq 0$ in $\left[V_{c}, V_{s}\right)$. Thus, from Lemma 7 ,

$$
-\frac{4}{5} V^{-1} W_{0} \kappa \leqq L_{1 / 3} \leqq 0 .
$$

In particular, in the incompressible case $\kappa=0$, Eq. (4.11) reduces to $\Sigma=L_{1 / 3}=0$ in $\left[V_{c}, V_{s}\right)$. Therefore, in this particular case, the second-order equation (3.4) can be reduced to the first-order equation $L_{1 / 3}=0$ which is readily integrated. This solution, i.e. the interior Schwarzschild solution, is the only one we know of having $\Sigma=0$ and satisfying our present criteria. Another asymptotically flat solution having $\Sigma=0$, due to Buchdahl, which has matter extending to infinity, is discussed in [9].

In the next section we will need an extension of $W_{0}(V)$ for values $V<V_{c}$. We define such an extension by requiring (compare [1])

$$
\Sigma(V)=0 \text { for } V<V_{c} \text {. }
$$


Lemma 8. $W_{0}(V)$ exists for all $V<V_{c}$ and is negative for $V>0$. Furthermore, $W_{0}$ is $C^{2}$ across $V=V_{c}$.

Proof. Existence and negativity are obvious from the form of $\Sigma$. The rest follows from $\Sigma\left(V_{c}\right)=\Sigma^{\prime}\left(V_{c}\right)=0$.

$W_{0}(V)$ does not, of course, satisfy Eq. (3.4) in the region $V<V_{c}$. Instead, we have, for all $V<V_{s}$,

$$
\begin{aligned}
W_{0} W_{0}^{\prime \prime}= & \frac{3}{4}\left(W_{0}^{\prime}\right)^{2}+\left[V^{-1} W_{0}-8 \pi V(\varrho+3 p)\right] W_{0}^{\prime}+8 \pi V W_{0}(\varrho+p)^{\prime} \\
& +16 \pi^{2} V^{2}(\varrho+3 p)^{2}+\frac{4}{5} W_{0}^{2} V^{-2} J
\end{aligned}
$$

where

$$
\begin{array}{lll}
J=0 & \text { for } & V \geqq V_{c}, \\
J=I & \text { for } & V<V_{c} .
\end{array}
$$

\section{The Main Theorem}

We now proceed to derive the divergence identities which are basic for the main theorem. These identities use as the dependent variable the quantity $W-W_{0}(V)$ where, as before $W=g^{a b} D_{a} V D_{b} V$ and $W_{0}(V)$ is the pull-back by the map $V: M \rightarrow \mathbf{R}$ of the function $W\left({ }^{0} V\right)$ corresponding to a spherical reference (SR-) model. In detail, we make the

SR-Assumption. There exists a spherical model $\left(\mathbf{R}^{3},{ }^{0} g_{a b},{ }^{0} V\right)$ with the same equation of state $\varrho(p)$ and the same surface potential $V_{s}$ as the given stellar model. We define $W_{0}(V)=W(V)$, where $W\left({ }^{0} V\right)={ }^{0} g^{a b} D_{a}^{0} V D_{b}^{0} V$.

We remark that it is not clear a priori that $V_{\min } \geqq V_{c}$ and for this reason we need the extension of $W_{0}$ below $V_{c}$ defined in the last section. The conditions on $\varrho(p)$, namely $\varrho>0, d \varrho / d p \geqq 0$ and $I \leqq 0$ will be required for all $p \in\left(0, \max \left(p_{\max }, p_{c}\right)\right]$.

The function $W_{0}$ satisfies, of course, $D_{a} W_{0}=W_{0}^{\prime} D_{a} V$ and

$$
\Delta \mathrm{W}_{0}=\mathrm{W} \mathrm{W}_{0}^{\prime \prime}+4 \pi \mathrm{V}(\varrho+3 \mathrm{p}) \mathrm{W}_{0}^{\prime} \text {. }
$$

To formulate a divergence-identity we perform the following conformal rescaling of $g_{a b}, W$ and $W_{0}$ on $Q$

$$
\tilde{\mathrm{g}}_{a b}=V^{-2} \Psi^{4} g_{a b}, \quad \tilde{W}=\Psi^{-4} W, \quad \tilde{W}_{0}=\Psi^{-4} W_{0} .
$$

Here $\Psi=\Psi(V)$ is defined, in the region $V \leqq V_{s}$, by

$$
\Psi^{-1} \Psi^{\prime}=\left(4 W_{0}\right)^{-1} L_{1 / 3} .
$$

Thereby $\Psi$ is determined up to a constant factor $\Psi\left(V_{s}\right)$ which we choose to be positive. We first note some properties of this function.

Lemma 9. $\Psi(V)>0$ for all $V \leqq V_{s}$. Furthermore, $\Psi(V)$ is $C^{\infty}$ for $V \neq V_{c}, C^{1}$ across $V=V_{c}$ and its second derivatives suffer a finite jump on $V=V_{c}$.

Proof. The positivity is obvious from Eq. (5.3). The rest follows by noting that, due to Lemma 8, $L_{1 / 3}$ is $C^{1}$ across $V=V_{c}$ and zero for $V=V_{c}$.

We remark that the second derivatives of $\Psi$ as a function on $M$ at $V=V_{c}$ can be defined as follows. Writing $D_{a} D_{b} \Psi=\Psi^{\prime} D_{a} D_{b} V+\Psi^{\prime \prime} D_{a} V D_{b} V, \Psi$ is $C^{2}$ at points of 
$V=V_{c}$ which are critical points of $V$. The non-critical points of $V=V_{c}$ form 2-surfaces on which $D_{a} D_{b} \Psi$ can be defined by taking the limit $V \rightarrow V_{c}\left(V>V_{c}\right)$. After a long computation, we obtain from Eq. (2.10), (4.13), (5.1), and (5.3),

$$
\begin{gathered}
{\left[\tilde{\Delta}-\frac{20 \pi}{3} V^{3} W_{0}^{-2} \Sigma(\varrho+3 p) \tilde{W}\right]\left(\tilde{W}-\tilde{W}_{0}\right)} \\
=\frac{1}{4} \tilde{W}^{-1} \widetilde{B}_{a b c} \tilde{B}^{a b c}+\frac{3}{4} \tilde{W}^{-1} \tilde{D}_{a}\left(\tilde{W}-\tilde{W}_{0}\right) \tilde{D}^{a}\left(\tilde{W}-\tilde{W}_{0}\right)-\frac{4}{5} \tilde{W}^{2} J \geqq 0
\end{gathered}
$$

in $Q$, where $\tilde{\Delta}$ and $\widetilde{B}_{a b c}$ are formed from $\tilde{g}_{a b}$. The inequality obviously holds provided $I \leqq 0$. In the special case $\varrho=$ const, we have $\Psi=$ const and $\Sigma=0$, and Eq. (5.4) is equivalent with Lindblom's [14] Eq. (19).

We have to comment on the singular terms in Eq. (5.4). Firstly, it can be shown, using $\Sigma\left(V_{c}\right)=\Sigma^{\prime}\left(V_{c}\right)=0$, that the quantity $W_{0}^{-2} \Sigma$ is finite at $V=V_{c}$ (but discontinuous). Furthermore, because of the terms containing $\tilde{W}^{-1}$ on the right-hand side, Eq. (5.4) still only makes sense at non-critical points $V$ in $Q$. But, due to $\Delta V>0$ in $Q$, the critical set is nowhere dense in $Q$. Thus, by using the inequality (5.4) off the critical set and taking limits, we obtain Eq. (5.4) everywhere in $Q$.

In the vacuum region $E$, we have to employ a more general equation than (the restriction of Eq. (5.4). Our choice is the two-parameter family of identities used by Robinson [12] in his uniqueness proof for static black holes. For our purpose, this family is conveniently written in terms of

$$
\bar{g}_{a b}=V^{-2} \Psi^{4} g_{a b}, \quad \bar{W}=\Psi^{-1}\left(1-V^{2}\right)^{-3} W, \quad \bar{W}_{0}=\Psi^{-1}\left(1-V^{2}\right)^{-3} W_{0},
$$

where $\Psi(V)$ is defined, in $E$, by

$$
\Psi=a\left(1-V^{2}\right)+b,
$$

and where $a$ and $b$ are constants. Here we have to specify these constants as follows:

$$
\begin{aligned}
& a=\frac{3}{32 \pi} m_{0}^{-2} \varrho(0)^{-1}\left(1-V_{s}^{2}\right)^{2}-\left(1-V_{s}^{2}\right)^{-1}, \quad b=1 \quad \text { if } \quad \varrho(0)>0 \\
& a=1, \quad b=0 \text { if } \varrho(0)=0,
\end{aligned}
$$

where $m_{0}$ denotes the mass of the SR-solution. When $\Psi(V)$ for $V \leqq V_{s}$ is the solution of Eq. (5.3) with $\Psi\left(V_{s}\right)=a\left(1-V_{s}^{2}\right)+b$, we obtain, as a complement to Lemma 9,

Lemma 10. $\Psi(V)>0$ for $V_{s} \leqq V<1$. Furthermore, $\Psi(V)$ is $C^{1}$ across $\partial Q$.

The proof is a simple verification. Using the variables introduced above, and $\Theta=\Psi\left(1-V^{2}\right)^{-1}$ Robinson's identity in $E$ reads,

$$
\begin{aligned}
\bar{\Delta}\left(\bar{W}-\bar{W}_{0}\right)= & \frac{1}{4} \bar{W}^{-1} \Theta^{6} \bar{B}_{a b c} \bar{B}^{a b c}+\frac{3}{4} \bar{W}^{-1} \Theta^{-2} \bar{D}_{a}\left[\Theta\left(\bar{W}-\bar{W}_{0}\right)\right] \\
& \times \bar{D}^{a}\left[\Theta\left(\bar{W}-\bar{W}_{0}\right)\right] \geqq 0 .
\end{aligned}
$$

We remark that, when the definition (5.3) and Eq. (5.4) are also used in the vacuum region, they become identical with Eqs. (5.6) and (5.8) in the case $\varrho(0)=0$.

Again, the inequality (5.8) is at first only valid off critical points of $V$ in $E$. But in $E$ the geometry $\left(g_{a b}, V\right)$ is well-known [24] to be analytic. The existence of an open critical set would imply $V=$ const in $E$ which would in turn contradict the 
positivity of the total mass. Thus the critical set is nowhere dense and we reason as before to obtain the inequality (5.8) everywhere in $E$.

We are now ready to show

Proposition 1. Assume we are given a static perfect-fluid model $\left(M, g_{a b}, V\right)$ with equation of state satisfying $I \leqq 0$, and a SR-solution $\left(\mathbf{R}^{3},{ }^{0} g_{a b},{ }^{0} V\right)$. Then $W-W_{0} \leqq 0$ and $V_{\min } \geqq V_{c}$.

Proof. We first consider the case where $\varrho(0)>0$. The maximum principle, when applied to $\bar{W}-\bar{W}_{0}$ in Eq. (5.8) yields the following result: Either $\bar{W}-\bar{W}_{0}$ takes on its maximum at infinity, where it vanishes. Or $\bar{W}-\bar{W}_{0}$ has a positive (absolute) maximum at some point $q \in \partial Q$. In the latter case, the boundary point lemma gives that $n^{a} D_{a}\left(\bar{W}-\bar{W}_{0}\right)<0$ at $q$, where the normal $n^{a}$ points inside $E$. But, on $\partial Q$,

$$
n^{a} D_{a}\left(\tilde{W}-\tilde{W}_{0}\right)=-6 \Theta^{-2} \Psi^{-2}\left(\bar{W}-\bar{W}_{0}\right) V n^{a} D_{a} V+\Theta^{-3} n^{a} D_{a}\left(\bar{W}-\bar{W}_{0}\right) .
$$

Due to $\bar{W}-\bar{W}_{0}>0$ at $q$ and $n^{a} D_{a} V>0$ on $\partial Q$, this implies

$$
\left.n^{a} D_{a}\left(\tilde{W}-\tilde{W}_{0}\right)\right|_{q}<0 .
$$

We now invoke inequality (5.4). Recall that $\Psi$ is $C^{2}$ except at non-critical points (w.r.t. $V$ ) of the set $V=V_{c}$. Since this set is clearly of measure zero, $\Psi$-and hence $\widetilde{W}-\widetilde{W}_{0}$ - are still in the space $W_{\text {loc }}^{2,3}(Q)$. Application of the weak maximum principle of Alexandrov (Theorem 9.1 in [16]) shows that

$$
\sup _{Q}\left(\tilde{W}-\tilde{W}_{0}\right) \leqq \max _{\partial Q}\left(\tilde{W}-\tilde{W}_{0}\right) \text {. }
$$

From Eq. (2.11) we see that $W-W_{0}$ and hence, due to Lemma $10, \tilde{W}-\tilde{W}_{0}$ is $C^{1}$ across $\partial Q$. But then Eq. (5.11) contradicts Eq. (5.10) when the point $q$ in the latter equation is chosen where $\tilde{W}-\widetilde{W}_{0}$ takes on its maximal value. Thus we are left with $\bar{W}-\bar{W}_{0} \leqq 0$ in $E$ which, together with Eq. (5.11) implies also $\sup _{Q}\left(\tilde{W}-\tilde{W}_{0}\right) \leqq 0$. This
settles the case $\varrho(0)>0$ in the proposition.

The argument in the case $\varrho(0)=0$ is formally simpler, but depends on a more detailed knowledge of the asymptotic behaviour of $\left(g_{a b}, V\right)$ near infinity. We first consider Eq. (5.8) in $E$. The definition of asymptotic flatness in Sect. 2 yields that $\bar{g}_{a b}$ extends to a smooth function on $E \cup\{\Lambda\}$. This fact, using the maximum principle on $E$, implies that either there is an absolute maximum of $\bar{W}-\bar{W}_{0}$ on $\partial Q$, or $\bar{W}-\bar{W}_{0}$ is constant in $E$. Assuming, in the first case, that the value of $\bar{W}-\bar{W}_{0}$ at this maximum is positive, we reach a contradiction in the same way as above. Next assume, in the second case, that $\bar{W}-\bar{W}_{0}=z_{0}>0$ in $E$. Note that this possibility was excluded in the case $\varrho(0)>0$, where we had (with a different definition of $\left.\bar{W}-\bar{W}_{0}\right) \lim _{\rightarrow \infty}\left(\bar{W}-\bar{W}_{0}\right)=0$. Now the Alexandrov maximum principle again gives $\sup \left(\tilde{W}-\tilde{W}_{0}\right) \leqq z_{0}$, and the strong maximum principle applied near $\partial Q$ shows that either $\tilde{W}-\widetilde{W}_{0}<z_{0}$ near $\partial Q$, or that $\tilde{W}-\widetilde{W}_{0}$ is constant also in $Q$. Since $\lim _{p \rightarrow 0} \kappa=\infty$, it can happen that $(\varrho+3 p) \sum$ diverges on $\partial Q$, which forbids the application of the boundary point lemma as given in Sect. 3.2 of [16]. [This is precisely the case where $\varrho^{\prime}(V)$ diverges at $V \rightarrow V_{s}^{<}$.] However, a suitably generalized version of this lemma, proven in the appendix, admits the present kind of singularity. When $\widetilde{W}-\widetilde{W}_{0}<z_{0}$ in $Q$ near $\partial Q$, Lemma A shows that again $n^{a} D_{a}\left(\tilde{W}-\widetilde{W}_{0}\right)>0$ on $\partial Q$. But this would contradict $\bar{W}-\bar{W}_{0}=z_{0}$ in $E$. Supposing now that $\widetilde{W}-\widetilde{W}_{0}=z_{0}=\bar{W}-\bar{W}_{0}$ everywhere, we infer, from Eq. (5.4), that $\Sigma=0$. This is again a contradiction since 
$\lim _{p \rightarrow 0} \kappa=\infty$, whereas the other terms in $\Sigma$ remain finite on $\partial Q$. (We remark that, if $p \rightarrow 0$

the star extended to infinity, this last conclusion could be evaded, since then $W_{0}$ would be zero where $\kappa$ diverges. The case $K=0$ provides an example [9].)

Thus we are only left with the possibility that $\bar{W}-\bar{W}_{0}$ in $E$ has a non-positive maximum on $\partial Q$. Invoking again the Alexandrov maximum principle shows that $W-W_{0} \leqq 0$ holds on $M$ for $\varrho(0)=0$ also.

Finally, $W-W_{0} \leqq 0$ implies $V_{\min } \geqq V_{c}$ since $W \geqq 0$ everywhere and $W_{0}<0$ for $V<V_{c}$.

We remark that from Proposition 1 there follows that $m$, the mass of the given solution, is not smaller than $m_{0}$, the mass of the SR-solution, since asymptotically, as $V \rightarrow 1$,

$$
0 \geqq W-W_{0} \sim \frac{1}{16}\left(m^{-2}-m_{0}^{-2}\right)\left(1-V^{2}\right)^{4} .
$$

One could perhaps argue on physical (e.g. stability) grounds that $m \leqq m_{0}$ implies $m=m_{0}$. If this is granted, the compactification argument used above in the case $\varrho(0)=0$ would imply the desired relation $W=W_{0}$. A mathematical proof is afforded by

Proposition 2. Under the requirements of Proposition 1, $W=W_{0}$ and $V_{\min }=V_{c}$.

Proof. Let ${ }^{0} \Omega:\left[V_{c}, 1\right] \rightarrow \mathbf{R}$ be the function introduced in Sect. 4 in the particular case of the present SR-solution and define $\Omega(V)={ }^{0} \Omega(V)$. Since $V \geqq V_{c}$, this is welldefined. A computation shows

$$
R\left[\Omega^{2} g\right]=2 \Omega^{-2}\left[2 \Omega^{-1} \Omega^{\prime \prime}-\Omega^{-2}\left(\Omega^{\prime}\right)^{2}\right]\left(W_{0}-W\right) .
$$

From Eq. (4.8), Lemma 6 and Proposition 1, we see that $R\left[\Omega^{2} g\right] \geqq 0$. Furthermore, from Eq. (4.7), $R\left[\Omega^{2} g\right]$ vanishes in $E$ and $\hat{g}_{a b}=\Omega^{2} g_{a b}$ has vanishing mass. Since $\hat{g}_{a b}$ is in $C^{1}(M) \cap C^{2}(\bar{Q}) \cap C^{2}(\bar{E})$, the assumptions for the positive-mass theorem to hold (see Bartnik [25]) are satisfied. It follows that $\left(M, g_{a b}\right)$ agrees with $\left(\mathbf{R}^{3}, \delta_{a b}\right)$. In particular, we have $R[\hat{g}]=0$.

Suppose now that the square bracket on the right in Eq. (5.13) was zero for all $V_{\min } \leqq V<V_{s}$. Integrating and using that $\Omega$ is $C^{1}$ across $\partial Q$, it follows that $\Omega(V)$ $=\frac{1}{4}(1+V)^{2}$ holds for all $V$. Inserting this into Eq. (4.8) gives $(1-V) \varrho=6 p V$. Taking the derivative w.r.t. $V$ of the latter equation, using Eq. (2.5) and integrating yields

$$
\varrho=\varrho_{0}(1-V)^{5}, \quad p=\frac{1}{6} \varrho_{0} V^{-1}(1-V)^{6},
$$

where $\varrho_{0}$ is a positive constant. Thus $p(V)$ does not go to zero for any $V_{s}<1$. This means that the square bracket in Eq. (5.13) cannot vanish identically. [This contradiction is evaded when $V_{s}=1$. In this case, Eq. (5.14) again gives rise to the equation of state employed by Buchdahl for which $K=0$.]

In order for $R[\hat{g}]$ to be zero we conclude that there are points in $Q$ where $W=W_{0}$. In particular, $W-W_{0}$ has a non-negative maximum in $Q$. Using again the maximum principle, this implies $W=W_{0}$ inside $Q$ and hence everywhere by the boundary point lemma.

Since $V$ attains its minimum in $Q, W$ must be zero somewhere. Since $W_{0}>0$ for $V>V_{c}$, it follows that $V_{\min }=V_{c}$. This ends the proof.

Combining Proposition 2 and Lemma 4, we obtain our main result.

Theorem. Under the requirements of Proposition 1, the given model and the SR-model are isometric. 
We finally point out that our result allows the following generalization to the $n$-body case (for $n=2$ compare Müller zum Hagen [26]). Suppose that $Q$ has $n$ connected components $Q^{i}(1 \leqq i \leqq n)$ with mutually disjoint smooth connected boundaries $\partial Q^{i}$ and identical boundary values $V_{s}$ of $V$ on $\partial Q^{i}$. Then we conclude as before that $W=W_{0}$. In particular $W>0$ in $E$ so that $V$ has no critical values for $V_{s} \leqq V<1$. Since the $V$-level sets are spheres near $V=1, V_{s}=$ const is also a topological sphere whence $\partial Q$ is connected so that $n=1$. Of course, the strong assumption on $V_{s}$ renders this result not satisfactory.

\section{Discussion}

In this section we first comment on the question of weakening the requirements of our theorem. Then there follows a discussion of some results on spherical symmetry of perfect fluids in Newton's theory.

There appear to be essentially two directions in which one could try to improve the uniqueness-result of this paper. One concerns the existence of the SR-model which is, of course, a highly implicit requirement. Once could try to actually prove this from the existence of the given model combined, presumably, with some bounds on $V_{s}$ depending on $\varrho(p)$. The other requirement one might try to weaken is $I \leqq 0$. The problem is that this condition enters the proof at four places: It controls the sign of $s$ in Eq. (4.8) (via $K \leqq 0$ ), the sign of $\Sigma$ in Eq. (5.4), the sign of $J$ in Eq. (5.4), and, for $\varrho(0)=0$, also the singular behaviour of $\Sigma$ [see Eq. (A.8)]. Improvements at the first two places seem possible upon strengthening Lemmas 6 and 7 respectively e.g. assuming $K \leqq 0$ or $I \leqq 0$ only for sufficiently large values of $p$. Next, the requirement on $\bar{J}$ in Eq. $(5.4)$ is related to the existence and extension of the SR-solution. One could remove $J$ from Eq. (5.4) altogether if, instead of solving Eq. (4.12) for $W_{0} \leqq 0$, a solution of Eq. (3.4) in this region was available. As regards the weakening of $I \leqq 0$ in Lemma A a comment is made there.

The main challenge is, however, giving a proof of spherical symmetry for any static perfect-fluid model satisfying the assumptions of Sect. 2-without taking the detour via uniqueness. It might be possible to employ the approach used here, combined with a better understanding of the spherical case, to complete this task also.

We now consider Newton's theory. The problem is to show spherical symmetry, respectively uniqueness, for solutions of $\Delta V=4 \pi \varrho$ and $D_{a} p=-\varrho D_{a} V$ in $\mathbf{R}^{3}$ for certain equations of state $\varrho(p)$. The other requirements of Sect. 2 apply with appropriate modifications (depending on whether one sets out to prove spherical symmetry or uniqueness as explained in Sect. 1 for the relativistic case). Here, however, direct proofs of spherical symmetry are available, which only require positivity of $p, \varrho$, and $d \varrho / d p \geqq 0$. In [27] Lichtenstein solved, under some relatively mild restrictions on the level sets of the density, a symmetry problem in hydrodynamics, which, in particular, yields spherical symmetry in the static case. Recalling that the present problem is equivalent to the study of the semilinear equation $\Delta V=4 \pi \varrho(V)$ on $\mathbf{R}^{3}$, we can, in the case where $\varrho\left(V_{s}\right)=0$, also invoke the general results of Gidas et al. [28] to conclude spherical symmetry. In either case, essential use is made of the symmetries of flat space and of the semi-linearity of the field equation, so it is by no means clear how to extend these methods to the relativistic case.

On the other hand, one could try to use the methods employed in this paper in the Newtonian case also. There, clearly, the positive-mass theorem is not available. 
Also, unlike in the relativistic case, the uniqueness of the solution is no longer related to conformal flatness of the 3-geometry. We can, however, adapt the basic formula of the present work, namely the elliptic equation (5.4) which leads to $W-W_{0} \leqq 0$. This step seems to be of independent interest since it is a wellestablished means of obtaining a-priori-bounds for the derivatives of solutions of elliptic or parabolic equations (see, e.g., Sperb [29]).

Using the same notation as for corresponding relativistic quantities we again introduce an SR-model, with $V_{c}={ }^{0} V_{\text {min }}$ and the same surface potential $V_{s}$ as the given one. We define

$$
\begin{gathered}
W=\delta^{a b} D_{a} V D_{b} V, \quad W_{0}\left({ }^{0} V\right)=\delta^{a b} D_{\alpha}^{0} V D_{b}^{0} V, \\
L_{1 / 3}=W_{0}^{\prime}-\frac{8 \pi}{3} \varrho \\
\Sigma=L_{1 / 3}+\frac{4}{5} W_{0} \frac{d \varrho}{d p} \\
I=\varrho \frac{d^{2} \varrho}{d p^{2}}+\frac{1}{5}\left(\frac{d \varrho}{d p}\right)^{2}, \\
B_{a b c}=4\left[\left(D_{a} D_{[b} V\right) D_{c]} V-\delta_{a[b} X_{c]}\right],
\end{gathered}
$$

where $X_{c}=\delta^{a b}\left(D_{a} D_{[b} V\right) D_{c]} V$,

$$
\Psi^{-1} \Psi^{\prime}=\left(4 W_{0}\right)^{-1} L_{1 / 3}
$$

and

$$
\tilde{\delta}_{a b}=\Psi^{4} \delta_{a b}, \quad \tilde{W}-\tilde{W}_{0}=\Psi^{-4}\left(W-W_{0}\right), \quad \tilde{B}_{a b c}=B_{a b c}
$$

in the domain $\left[V_{c}, V_{s}\right.$ ), extend $W_{0}$ to $V \leqq V_{c}$ as before and define $J$ as in Eq. (4.14). We obtain, as analogue of Eq. (5.4),

$$
\begin{aligned}
& {\left[\tilde{\Delta}-\frac{20 \pi}{3} W_{0}^{-2} \Sigma \varrho \tilde{W}\right]\left(\tilde{W}-\tilde{W}_{0}\right)} \\
& \quad=\frac{1}{4} \tilde{W}^{-1} \widetilde{B}_{a b c} \widetilde{B}^{a b c}+\frac{3}{4} \tilde{W}^{-1} \tilde{D}_{a}\left(\tilde{W}-\tilde{W}_{0}\right) \tilde{D}^{a}\left(\tilde{W}-\tilde{W}_{0}\right)-\frac{4}{5} \tilde{W}^{-2} J .
\end{aligned}
$$

In the vacuum region, there also exists an analogue of Eq. (5.8) [which again coincides with the restriction of Eq. (6.8) in the case $\varrho(0)=0]$. The correspondence between the object $B_{a b c}$ as defined in Eq. (6.5) and the "Cotton-tensor" (Eq. 2.9) can be made manifest by inserting the field equations $(2.2,3)$ into the latter. We also remark that spherical symmetry is again characterized by $W=W_{0}$ (due to an analogue of Lemma 4) or, alternatively, by the condition $B_{a b c}=0$.

In the relativistic case we showed that, for matter satisfying the Buchdahl equation $p=\frac{1}{6} \varrho^{6 / 5}\left(\varrho_{0}^{1 / 5}-\varrho^{1 / 5}\right)^{-1},\left(\varrho_{0}=\right.$ const $)$, uniqueness follows directly from $W-W_{0} \leqq 0$ [9]. In the present situation we similarly obtain the same result for $p=$ const $\varrho^{6 / 5}$, the polytrope of index 5 (which is the non-relativistic limit of the Buchdahl condition). In general, if the equation of state satisfies $I \leqq 0$, we arrive, as before, at the conclusion that $W-W_{0} \leqq 0$.

In the case where $\varrho=$ const, we are able to complete this result to a uniqueness proof. The idea is to modify the first step of Weinberger's proof [30] of a certain "overdetermined boundary value problem" for $\Delta V=$ const [his second step just consists of using (6.8) for $\Sigma=I=0$ and $\Psi=$ const to show that $\left.W-W_{0} \leqq 0\right]$. It would be of interest to find an argument corresponding to Proposition 2 for 
general equations of state (satisfying $I \leqq 0$ ). This should then also give hints for modifying, and perhaps improving, the relativistic proof.

Acknowledgements. We thank P. Michor and B. Schmidt for helpful remarks.

\section{Appendix}

We derive here a version of the boundary-point lemma in a situation where the zeroth-order term diverges on the boundary $\partial Q$, but which requires a bit more smoothness of the other coefficients of the differential operator on $\partial Q$ and of $\partial Q$ itself than the standard one. In this lemma we refer, for convenience, to the set $Q$ and the function $V$ as defined in Sect. 2. It is, however, only important that given a domain $Q$, there exists a $C^{2}$-function on $\bar{Q}$ near $\partial Q$, which is constant and free of critical points on $\partial Q$. Hence Lemma A could be formulated and proven without any reference to the particular situation at hand.

Lemma A. Consider a neighbourhood $Q^{\prime}$ of $\partial Q$ in $Q$ of the form

$$
Q^{\prime}=\left\{x \in Q \mid V^{\prime}<V(x)<V_{s}\right\} .
$$

$V^{\prime}$ is chosen such that there are no critical values of $V$ for $V^{\prime} \leqq V \leqq V_{s}$. Suppose there is a function $u \in C^{2}\left(Q^{\prime}\right) \cap C^{1}\left(\bar{Q}^{\prime}\right)$ which satisfies

$$
L u=\left(\Delta+k^{a} D_{a}+c\right) u \geqq 0
$$

in $Q^{\prime}$, where $g_{a b} \in C^{1}\left(\bar{Q}^{\prime}\right), k^{a} \in C^{0}\left(\bar{Q}^{\prime}\right)$ and $c$ is non-positive in $Q^{\prime}$ and of the form $c=d+e f^{-1}$. Here $d$, e are $C^{0}\left(\bar{Q}^{\prime}\right)$ and $f$ is $C^{0}\left(\bar{Q}^{\prime}\right) \cap C^{1}\left(Q^{\prime}\right)$, with $f \neq 0$ in $Q^{\prime}$ and $f=0$ on $\partial Q$. Furthermore, $\lim _{\rightarrow \partial Q} n^{a} D_{a} f=F$, where $F$ is continuous, possibly infinite, but nonvanishing on $\partial Q$, and where the $V$-level sets carry the unit-normal $n^{a}$ (directed towards increasing $V$ ) along the integral curves of which the lim is taken. Finally, assume that there is a point $x_{0} \in \partial Q$ such that

$$
u(x)<u\left(x_{0}\right)=u_{0} \geqq 0
$$

for all $x \in Q^{\prime}$. Then we have

$$
\left.n^{a} D_{a} u\right|_{x_{0}}>0
$$

Proof. Define a quantity $v$ by

$$
v=\exp (-\alpha V)-\exp \left(-\alpha V_{s}\right),
$$

where the constant $\alpha$ is disposed of later. Using l'Hospital's rule, we find

$$
\lim _{\rightarrow \partial Q} f^{-1} v=-\left.\alpha \exp \left(-\alpha V_{s}\right) F^{-1} W^{1 / 2}\right|_{\partial Q} .
$$

Thus $L v$ admits a continuous extension to $\partial Q$, namely

$$
\left.\exp \left(\alpha V_{s}\right) L v\right|_{\partial Q}=\left.\left(\alpha^{2} W-\alpha \Delta V-\alpha k^{a} D_{a} V-\alpha e F^{-1} W^{1 / 2}\right)\right|_{\partial Q} .
$$

Since $W>0$ on $\partial Q$, we can choose $\alpha$ large enough such that $L v>0$ on $\partial Q$. Choosing, if necessary $V^{\prime}$ still closer to $V_{s}$, we have $L v \geqq 0$ throughout $Q^{\prime}$. By virtue of (A.2) we have $u<u_{0}$ on the inner boundary $V=V^{\prime}$. Hence there is a constant $\varepsilon>0$ such that $u-u_{0}+\varepsilon v \leqq 0$ on $V=V^{\prime}$. Since $v$ vanishes on $\partial Q$ and since, by continuity, $u-u_{0} \leqq 0$ on $\partial Q$, we also have $u-u_{0}+\varepsilon v \leqq 0$ on $\partial Q$. Furthermore $L\left(u-u_{0}+\varepsilon v\right)$ 
$\geqq-c u_{0} \geqq 0$ in $Q^{\prime}$. Thus, from the weak maximum principle (Corollary 3.2 of [16]), it follows that $u-u_{0}+\varepsilon v \leqq 0$ in all of $Q^{\prime}$. Consequently, using $\left(u-u_{0}+\varepsilon v\right)\left(x_{0}\right)=0$, we find that $n^{a} D_{a}(u+\varepsilon v) \geqq 0$ at $x_{0}$, which implies

$$
\left.n^{a} D_{a} u\right|_{x_{0}} \geqq-\left.\varepsilon n^{a} D_{a} v\right|_{x_{0}}=\left.\varepsilon \alpha \exp \left(-\alpha V_{s}\right) W^{1 / 2}\right|_{x_{0}}>0 .
$$

The proof of Lemma $\mathrm{A}$ is complete.

It remains to see whether Lemma A can be applied to Eq. (5.4). Transforming this equation back to the original metric $g_{a b}$, but keeping $u=\widetilde{W}-\widetilde{W}_{0}$, Eq. (5.4) can clearly be brought into the form of Eq. (A.1) when we choose $f(V)=(\varrho+3 p)^{-\delta} \lambda$, where $\lambda=\kappa^{-1}$ as before and $\delta=$ const $\leqq 1$. Note that the factor $(\varrho+3 p)^{1-\delta}$ is absorbed into $e$. In the case of present interest $\varrho, p$, and $\lambda$ all vanish on $\partial Q$. If $f$ does not go to zero on $\partial Q, e \cdot f^{-1}$ remains bounded so that the usual form of the Hopf lemma applies. Suppose now that $f\left(V_{s}\right)$ is zero. Using $I \leqq 0$ we find

$$
\frac{d f}{d V} \leqq(\varrho+3 p)^{-\delta} V^{-1}\left[3 \delta(\varrho+3 p)^{-1}(\varrho+p) \lambda-2 \lambda+\delta-\frac{1}{5}\right] .
$$

Choosing $0<\delta<1 / 5$ it follows that

$$
\lim _{\rightarrow \partial Q} n^{a} D_{a} f=\lim _{V \rightarrow V_{s}} W^{1 / 2}(d f / d V)=-\infty
$$

and Lemma A applies.

We remark that $I \leqq 0$ is used here only to have some control over the second derivatives of $\varrho(p)$ near $p=0$. If we had e.g. $I \geqq 0$ near $p=0$, the proof would also go through.

\section{References}

1. Masood-ul-Alam, A.K.M.: A proof of uniqueness of static stellar models with small $d \varrho / d p$. Class. Quantum Grav. 5, 409-421 (1988)

2. Künzle, H.P., Savage, J.R.: A global analysis approach to the general relativistic fluid ball problem. Gen. Rel. Grav. 12, 155-174 (1980)

3. Schmidt, B.G.: Kugelsymmetrische statische Materielösungen der Einsteinschen Feldgleichungen. Diploma thesis. Hamburg 1966

4. Shapiro, S.L., Teukolsky, S.A.: Black holes, white dwarfs, and neutron stars. New York, Chichester, Brisbane, Toronto, Singapore: Wiley 1983

5. Harrison, B.K., Thorne, K.S., Wakano, M., Wheeler, J.A.: Gravitation theory and gravitational collapse. Chicago, London: The University of Chicago Press 1965

6. Schmid, W.: Numerische Integration der Tolman-Oppenheimer-Volkov Gleichungen. University of Vienna 1972 (unpublished)

7. Schoen, R., Yau, S.-T.: Proof of the positive mass theorem. Commun. Math. Phys. 65, 45-76 (1979)

8. Bunting, G.L., Masood-ul-Alam, A.K.M.: Nonexistence of multiple black holes in asymptotically Euclidean static vacuum space-time. Gen. Rel. Grav. 19, 147-154 (1987)

9. Beig, R., Simon, W.: On the spherical symmetry of static perfect fluids in general relativity. Lett. Math. Phys. 21, 245-250 (1991)

10. Buchdahl, H.A.: A relativistic fluid sphere resembling the Emden polytrope of index 5. Astrophys. J. 140, 1512-1516 (1964)

11. Israel, W.: Event horizons in static vacuum space-times. Phys. Rev. 164, 1776-1779 (1967)

12. Robinson, D.C.: A simple proof of the generalization of Israel's theorem. Gen. Rel. Grav. 8, 695-698 (1977)

13. Lindblom, L.: Some properties of static general relativistic stellar models. J. Math. Phys. 21, 1455-1459 (1980) 
14. Lindblom, L.: Static uniform-density stars must be spherical in general relativity. J. Math. Phys. 29, 436-439 (1988)

15. Oppenheimer, J.R., Volkoff, G.M.: On massive neutron cores. Phys. Rev. 55, 374-381 (1939)

16. Gilbarg, D., Trudinger, N.S.: Elliptic partial differential equations of second order. Berlin, Heidelberg, New York: Springer 1983

17. Künzle, H.P.: On the spherical symmetry of a static perfect fluid. Commun. Math. Phys. 20, 85-100 (1971)

18. Avez, A.: Le $d s^{2}$ de Schwarzschild parmi les $d s^{2}$ stationnaires. Ann. Inst. Henri Poincaré 1, 291-300 (1964)

19. Lichnerowicz, A.: Théories relativistes de la gravitation et de l'électromagnétisme. Paris: Masson 1955

20. Beig, R., Simon, W.: Proof of a multipole conjecture due to Geroch. Commun. Math. Phys. 78, 75-82 (1980)

21. Beig, R.: Conformal properties of static spacetimes. Class. Quantum Grav. 8, 263-271 (1991)

22. Simon, W., Beig, R.: The multipole structure of stationary space-times. J. Math. Phys. 24, 1163-1171 (1984)

23. Beig, R.: A remarkable property of spherical harmonics. J. Math. Phys. 26, 769-770 (1985)

24. Müller zum Hagen, H.: On the analyticity of static solutions of Einstein's equations. Proc. Camb. Phil. Soc. 67, 415-421 (1970)

25. Bartnik, R.: The mass of an asymptotically flat manifold. Commun. Pure Appl. Math. 49, 661-693 (1986)

26. Müller zum Hagen, H.: The static two body problem. Proc. Camb. Phil. Soc. 75, 249-260 (1974)

27. Lichtenstein, L.: Über einige Eigenschaften der Gleichgewichtsfiguren rotierender Flüssigkeiten, deren Teilchen einander nach dem Newtonschen Gesetze anziehen. Math. Z. 28, 635-640 (1928)

28. Gidas, B., Ni, W.-M., Nirenberg, L.: Symmetry of positive solutions of nonlinear elliptic equations in $\mathbf{R}^{n}$. Adv. Math. Suppl. Stud. 7A, 369-402 (1981)

29. Sperb, R.P.: Maximum principles and their applications. New York, London, Toronto, Sydney, San Francisco: Academic Press 1981

30. Weinberger, H.F.: Remark on the preceding paper of Serrin. Arch. Rational Mech. Anal. 43, 319-320 (1971)

Communicated by S.-T. Yau 